\title{
STOCHASTIC TECHNIQUES FOR THE NUMERICAL SOLUTION OF ENGINEERING BOUNDARY VALUE PROBLEMS.
}

\author{
V. Maceiras and M. Casteleiro \\ Group of Numerical Methods in Engineering \\ E.T.S. de Ingenieros de Caminos, Canales y Puertos \\ Universitity of Coruña, Spain \\ e-mail: victormaceiras@hotmail.com, casteleiro@udc.es
}

Keywords: Stochastic analysis, PDE solutions, Random walk, Itô's theorem.

Abstract. This paper describes a family of stochastic methods based on Itô's theorem for the numerical solution of Dirichlet, Neumann and mixed boundary value problems. The major advantages of these techniques are:

- No mesh is needed. Only the geometry of the domain and the boundary conditions should be defined.

- The solution can be obtained at any point or at any part of the integration domain. It is not necessary to solve the whole domain, which can be of special interest in $3 D$ applications.

- The error threshold can be controlled mainly with the step width of the random walk generation

- The algorithms can be easily parallelized.

- The same approach could be used to the numerical solution of stochastic partial differential equations.

The theoretical approach is described, the corresponding algorithms are presented, and the general technique is applied to well-known engineering PDE equations of the elliptic and parabolic type. 


\section{INTRODUCTION}

From the origin of calculus in the 17 th century by Isaac Newton and Gottfried Leibniz partial differential equations have been used to generate mathematical models of natural, economic and social phenomena. In general, these equations cannot be solved by analytical methods and, from the beginning of the 20th century, different approaches generating several discretization approximations have been developed (i.e. Ritz methods [1]). Since then a number of numerical methods, as Finite Differences, Finite Element Method, Boundary Element Method, Finite Calculus Techniques and many others have proved to give accurate results for quite difficult problems, both linear and nonlinear, in one, two and three dimensions.

In general, all these techniques imply some type of domain decomposition or discretization, and the solution of the problem must be obtained in the whole integration domain. As a consequence of the discretization, a set of algebraic equations (linear or nonlinear) must be solved.

A different approach to the solution of PDE's is presented in this paper. Probabilistic methods that have been developed from 1940 [2] may be used to solve both deterministic and stochastic differential equation by means of simulation techniques.

One of these possible techniques, based in Brownian motion particle simulation will be shown to be applied to elliptic and parabolic partial differential equations.

The advantages of this technique are:

- No mesh is needed. Only the geometry of the domain and the boundary conditions should be defined. This implies that the time-consuming discretization process in unnecessary.

- As it is not necessary to get the solution at every point of the domain, no system of equations must be solved.

- The solution can be obtained at any point or at any part of the integration domain. It is not necessary to determine the solution at the whole domain, and therefore there is not system of equations to solve, which can be of special interest in $3 \mathrm{D}$ and nonlinear problems.

- The error threshold can be controlled mainly with the step width of the random walk generation

- The algorithms can be easily parallelized.

- The same approach could be used to the numerical solution of stochastic partial differential equations [3].

In this paper the general technique for elliptic and parabolic differential equations will be presented, and will be applied to benchmark problems whose analytical solution is known.

\section{PREVIOUS DEFINITIONS}

\subsection{Wiener processes.}

A real stochastic process $\left\{\mathrm{W}_{\mathrm{t}}, \mathrm{t} \geq 0\right\}$ is called a Wiener process if all the following requirements are accomplished:

- $\mathrm{W}_{0}=0$

- $\mathrm{W}_{\mathrm{t}}-\mathrm{W}_{\mathrm{s}}$ is a random normal variable $\mathrm{N}(0, \mathrm{t}-\mathrm{s})$ for every $\mathrm{t}$ and $\mathrm{s}$ such that $\mathrm{t}>\mathrm{s} \geq 0$

- For every set of arguments $0<\mathrm{t}_{1}<\mathrm{t}_{2}<\cdots<\mathrm{t}_{\mathrm{n}}$, the set of random variables $\mathrm{W}_{\mathrm{t}_{1}}, \mathrm{~W}_{\mathrm{t}_{12}}-\mathrm{W}_{\mathrm{t}_{1}}, \ldots . \mathrm{W}_{\mathrm{t}_{\mathrm{n}}}-\mathrm{W}_{\mathrm{t}_{-1}}$ are independent.

- $\mathrm{W}_{\mathrm{t}}$ has continuous paths for every $\mathrm{t}>0$. 


\subsection{Martigales}

Consider a sequences of random variables $Z_{1}, Z_{2} \ldots . Z_{n}$ and the conditional expected value $E\left[Z_{n+1} \mid Z_{1}, Z_{2} \ldots . Z_{n}\right]$ for each $n$. The conditional expected value is the mean of $Z_{n+1}$ if all values $Z_{1}, Z_{2} \ldots \ldots Z_{n}$ are known. If $E\left[Z_{n+1} \mid Z_{1}, Z_{2} \ldots \ldots Z_{n}\right]=Z_{n}$ the sequence, $Z_{2} \ldots \ldots Z_{n}$ is a martingale.

The Wiener process is a Markov process as well as a martingale.

\subsection{Itô's process}

The stochastic process

$$
d X=b(X(t), t) d t+\sigma(X(t), t) d B
$$

in which $d B$ is a standard Brownian motion (BM), is called an Itô's process and $b(X(t), t)$ and $\sigma(X(t), t)$ are functions both of time and the stochastic process $X(t)$. [4, 5]

\subsection{Standard Brownian motion}

A standard Brownian motion $B(t)$ is a Wiener process such that:

- $E[B(t)]=0$

- $\Delta B=B(t+\Delta t)-B(t)$ has a probability distribution $N\left(0, s^{2}\right)$

Therefore $\operatorname{Var}(\Delta B)=\Delta t=E\left(\Delta B^{2}\right)-(E(\Delta B))^{2}=E\left(\Delta B^{2}\right)$ and as the variance of $\Delta B$ is small it can be assumed that $\Delta t \approx \Delta B^{2}$. [6, 7]

\subsection{Stochastic ordinary differential equations}

An ordinary differential equation is only a rule which determines the change of a dependent variable which is a function of an independent variable expressed in terms of the function and its derivatives.

$$
\frac{d X}{d t}=a(t, X)
$$

If non deterministic parameters appear in the differential system, the equation becomes a stochastic differential equation

$$
\frac{d X}{d t}=a(t, X, Y)
$$

where $Y(t)$ represents a stochastic process. It is obvious that in this case $X(t)$ is also a stochastic process and the solution will be the given by the probability distribution of $X(t)$.

\subsection{Itô's integral}

If one cannot integrate $a(t, X, Y)$ the stochastic ordinary differential equation can be rewritten as

$$
\frac{d X}{d t}=b(X, t)+\sigma(X, t) \frac{d B(t)}{d t}
$$

where $d B(t)$ is a standard brownian motion. The integral of the above equation is

$$
X(t)=X_{0}+\int_{t_{0}}^{t} b(s, X(s)) d s+\int_{t_{0}}^{t} \sigma(s, X(s)) d B(t)
$$

This second integral $\int_{t_{0}}^{t} b(s, X(s)) d B(t)$ cannot be univocally defined, because $\mathrm{W}(\mathrm{s})$ variations are unbounded, given that $\sup \sum_{\mathrm{k}=1}^{\mathrm{n}-1}\left|\mathrm{~B}_{\mathrm{t}_{\mathrm{k}+1}}-\mathrm{B}_{\mathrm{t}_{\mathrm{k}}}\right|=\infty$. The most correct definition 
from a mathematical point of view was established by Itô in the forties and was the origin of the so called stochastic analysis [4, 5, 8, 9].

The most important property of Itô's integral is $E\left(\int_{0}^{T} G d B\right)=0[10,11]$.

\subsection{Itô's formula}

Let $u=u(t, \bar{x})$ be a continuous function defined over $\left[t_{0}, T\right] \times \mathbb{R}^{d}$ taking values in $\mathbb{R}^{d}$ and with continuous partial derivatives. We will use the following notation:

$$
\begin{gathered}
\frac{\partial}{\partial t} u(t, \bar{x})=u_{t}(t, \bar{x}) \\
\frac{\partial}{\partial x} u(t, \bar{x})=u_{x}(t, \bar{x}) \\
\frac{\partial^{2}}{\partial x^{2}} u(t, \bar{x})=u_{x^{2}}(t, \bar{x})
\end{gathered}
$$

For $i, j=1 \ldots . d$ let $\left\{X, t_{0} \leq t \leq \mathrm{T}\right\}$ be a stochastic process in $\mathbb{R}^{d}$. This process is defined by the following stochastic differential (Itô's process)

$$
d X=b(X(t), t) d t+\sigma(X(t), t) d B
$$

where $d B$ is a standard Brownian motion. Then, the process defined by

$$
Y(t)=u(t, X(t)), \forall t \in\left[t_{0}, \mathrm{~T}\right]
$$

can be differentiated with respect the same Brownian motion

$$
\begin{gathered}
d Y=u_{t}\left(t, X_{t}\right) d t+u_{x}\left(t, X_{t}\right) d X+\frac{1}{2} u_{x^{2}}\left(t, X_{t}\right) \sigma^{2} d t= \\
=\left(u_{t}\left(t, X_{t}\right)+u_{x}\left(t, X_{t}\right) b(X(t), t)+\frac{1}{2} u_{x^{2}}\left(t, X_{t}\right) \sigma^{2}\right) d t+u_{x}\left(t, X_{t}\right) \sigma(X(t), t) d B
\end{gathered}
$$

To prove this result we apply Taylor's expansion to $u$,

$$
d u=\frac{\partial u}{\partial X} \Delta X+\frac{\partial u}{\partial t} \Delta t+\frac{1}{2} \frac{\partial^{2} u}{\partial X^{2}} \Delta X^{2}+\cdots
$$

Itô's process can be rewritten as:

$$
\begin{gathered}
d X=b(X(t), t) d t+\sigma(X(t), t) \sqrt{d t} \\
(d X)^{2}=(b(X(t), t))^{2} d t^{2}+(\sigma(X(t), t))^{2} d t+2 b(X(t), t) d t \sigma(X(t), t) \sqrt{d t} \\
(d X)^{2}=(\sigma(X(t), t))^{2} d t
\end{gathered}
$$

By replacing $d X$ y $(d X)^{2}$ in the above Taylor expansion expression

$$
\begin{aligned}
& d u=\frac{\partial u}{\partial X}(b(X(t), t) d t+\sigma(X(t), t) d B)+\frac{\partial u}{\partial t} \Delta t+\frac{1}{2} \frac{\partial^{2} u}{\partial X^{2}}(\sigma(X(t), t))^{2} d t \\
& d u=\frac{\partial u}{\partial X} \sigma(X(t), t) d B+d t\left(b(X(t), t) \frac{\partial u}{\partial X}+\frac{\partial u}{\partial t}+\frac{1}{2} \frac{\partial^{2} u}{\partial X^{2}}(\sigma(X(t), t))^{2}\right)
\end{aligned}
$$

Which is known as Itô's formula.

\section{ELLIPTIC PROBLEM GENERAL APPROACH}

Let be the following elliptic partial differential equation in one spatial dimension 


$$
\mathrm{L} u=-f(x)
$$

with Dirichlet boundary condition

$$
u(x)=g(x) \text { si } x \in \partial D
$$

being $\partial D$ the boundary of the integration domain $D$ and $\mathrm{L}($.

$$
\mathrm{L}(.)=\sum b_{i} \frac{\partial(.)}{\partial x_{i}}+\frac{1}{2} \sum \sum \sigma_{i j} \frac{\partial^{2}}{\partial x_{i} \partial x_{j}}
$$

that will be applied to a function $u(t, X(t))$. The $d u$ differential can be written as

$$
d u=\frac{\partial u}{\partial X} \sigma(X(t), t) d B+d t\left(b(X(t), t) \frac{\partial u}{\partial X}+\frac{\partial u}{\partial t}+\frac{1}{2} \frac{\partial^{2} u}{\partial X^{2}}(\sigma(X(t), t))^{2}\right)
$$

As the problem is steady state by definition

$$
\frac{\partial u}{\partial t}=0
$$

and therefore

$$
d u=\frac{\partial u}{\partial X} \sigma(X(t), t) d B+d t(L(u))
$$

or

$$
d u=\frac{\partial u}{\partial X} \sigma(X(t), t) d B-(f(x)) d t
$$

This equation can be integrated as

$$
\int_{0}^{\tau_{x}} d u=\int_{0}^{\tau_{x}} \frac{\partial u}{\partial X} \sigma(X(t), t) d B-\int_{0}^{\tau_{x}}(f(x)) d t
$$

being $\tau_{x}$ the so called stopping time, which is defined as the shortest time in which the stochastic process $X$ reaches the boundary $\partial D$,

$$
\tau_{x}=\inf \left(\frac{t}{X} \in \partial D\right)
$$

and consequently

$$
u\left(X^{\tau_{x}}\right)-u\left(X^{0}\right)=\int_{0}^{\tau_{x}} \frac{\partial u}{\partial X} \sigma(X(t), t) d B-\int_{0}^{\tau_{x}}(f(x)) d t
$$

The expected value of these expressions is

$$
E\left(u\left(X^{\tau_{x}}\right)\right)-E\left(u\left(X^{0}\right)\right)=E\left(\int_{0}^{\tau_{x}} \frac{\partial u}{\partial X} \sigma(X(t), t) d B\right)-E\left(\int_{0}^{\tau_{x}}(f(x)) d t\right)
$$

As we have defined the stopping time $\tau_{x}$ as the shortest time in which the stochastic process $X$ reaches the boundary $\partial D$, if $u\left(X^{0}\right)$ is the value of $u(x)$ to obtain inside the integration domain,

$$
E\left(g\left(X^{\tau_{x}}\right)\right)-u(x)=E\left(\int_{0}^{\tau_{x}} \frac{\partial u}{\partial X} \sigma(X(t), t) d B\right)-E\left(\int_{0}^{\tau_{x}}(f(x)) d t\right)
$$


where $g\left(X^{\tau_{x}}\right)$ is the boundary condition at the point in which the stochastic process $X$ reaches the boundary $\partial D$. Remember that $d B$ is a martingale and one of its properties is $E\left(\int_{0}^{T} G d B\right)=0$.

Therefore

$$
E\left(g\left(X^{\tau_{x}}\right)\right)-u(x)=0-E\left(\int_{0}^{\tau_{x}}(f(x)) d t\right)
$$

and the Itô's formula becomes

$$
u(x)=E\left(g\left(X^{\tau_{x}}\right)\right)+E\left(\int_{0}^{\tau_{x}}(f(x)) d t\right)
$$

\subsection{Problem solution in several variables}

In the case of more than one variable the elliptic problem can be stated as

$$
\begin{gathered}
\mathrm{Lu}=-f(\bar{x}) \\
u(\bar{x})=g(\bar{x}) \text { si } \bar{x} \in \partial D \\
\mathrm{~L}(.)=\sum b_{i} \frac{\partial(.)}{\partial x_{i}}+\frac{1}{2} \sum \sum \sigma_{i j} \frac{\partial^{2}}{\partial x_{i} \partial x_{j}}
\end{gathered}
$$

The corresponding stochastic process could be written as

$$
d \bar{X}=\bar{b}(\bar{x})) d t+\overline{\bar{Z}}(\bar{x})) d \bar{B}
$$

where $b_{i}$ are the components of vector $\left.\bar{b}(\bar{x})\right)$.

The matrix $\overline{\bar{Z}}(x)$ is defined as follows [12]

$$
\overline{\bar{C}}=\overline{\bar{Z}} * \overline{\bar{Z}}^{T}
$$

where $\sigma_{i j}$ are the components of the $\overline{\bar{C}}$ matrix. This matrix $\overline{\bar{Z}}$ can be easily determined if $\overline{\bar{C}}$ is symmetric, by using, for example, the Cholesky factorization technique.

The $b_{i}$ and $\sigma_{i j}$ components are the parameters of the differential operator L(.).

\subsection{Problems with Neumann boundary conditions}

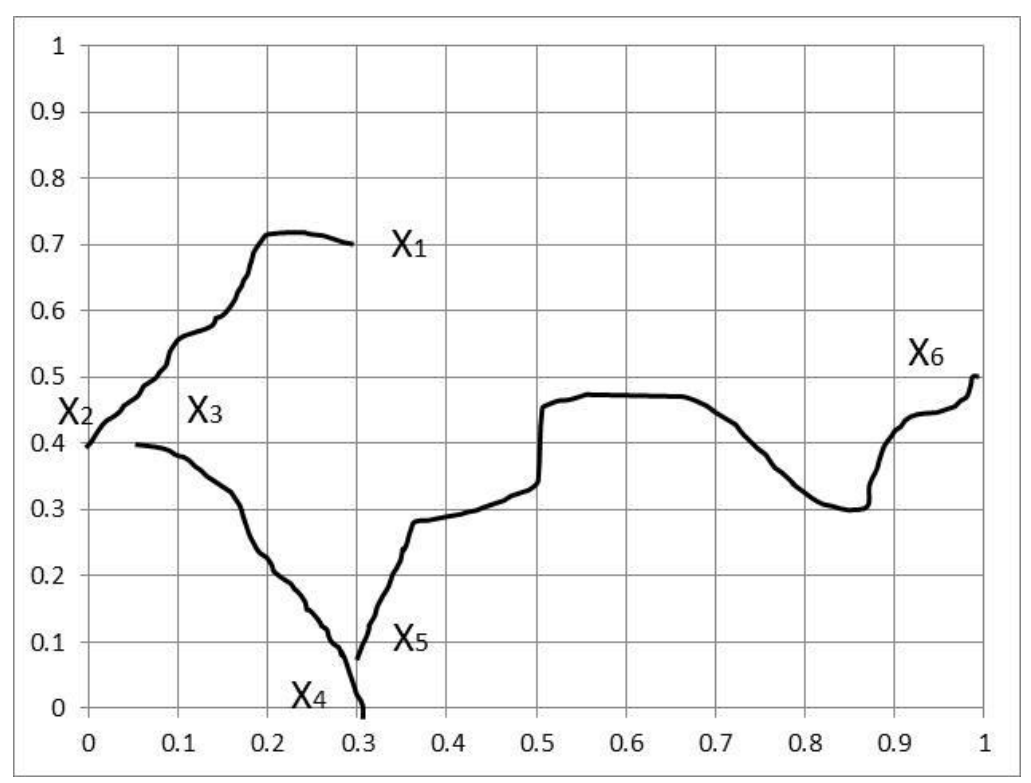

Figure 1 Particle random walk 
It must be noted that every particle follows a random walk determined by the Itô's stochastic process (Figure 1).

But in this case there are some Neumann boundary conditions. We describe now the procedure to take into account these boundaries. [13].

In a general case with Neumann boundary conditions and at least a Dirichlet boundary condition the procedure will be as follows:

$$
\begin{gathered}
\mathrm{Lu}=-f(\bar{x}) \\
u(\bar{x})=g(\bar{x}) \text { si } \bar{x} \in \partial D_{d} \\
\frac{\partial(u(\bar{x}))}{\partial n}=c(\bar{x}) \text { si } \bar{x} \in \partial D_{n}
\end{gathered}
$$

where $\partial D_{d}$ are the boundaries in which Dirichlet conditions hold and $\partial \mathrm{D}_{\mathrm{n}}$ the boundaries in which Neumann conditions are defined.

One should determine $X_{1}$ when the particle reaches a Neumann boundary before getting to a Dirichlet boundary.

$$
u\left(X_{5}\right)=E\left(g\left(X^{\tau_{x}}\right)\right)+E\left(\int_{0}^{\tau_{x}}(f(x)) d t\right)=E\left(g\left(X_{6}\right)\right)+E\left(\int_{\tau_{X_{5}}}^{\tau_{X_{6}}}(f(x)) d t\right)
$$

The integration limits $\left[\tau_{X_{5}}, \tau_{X_{6}}\right]$ correspond to the time the particle takes to move from $X_{5}$ to $X_{6}$

If the coordinates $X_{4}$ correspond to a point located in a boundary with Neumann condition, the value $u\left(X_{4}\right)$ may be determined by Taylor expansion as

$$
u\left(X_{4}\right)=u\left(X_{5}\right)-\frac{\partial u}{\partial y}\left(X_{4}\right) h_{y}=u\left(X_{5}\right)-c\left(X_{4}\right) h_{y}
$$

In this way, the Neumann condition in $X_{4}$ is transformed to a Dirichlet condition and therefore:

$$
\begin{gathered}
u\left(X_{3}\right)=E\left(g\left(X^{\tau_{x}}\right)\right)+E\left(\int_{0}^{\tau_{x}}(f(x)) d t\right)=E\left(u\left(X_{4}\right)\right)+E\left(\int_{\tau_{X_{4}}}^{\tau_{X_{5}}}(f(x)) d t\right)= \\
=E\left(u\left(X_{5}\right)\right)-E\left(c\left(X_{4}\right) h_{y}\right)+E\left(\int_{\tau_{X_{4}}}^{\tau_{X_{5}}}(f(x)) d t\right)= \\
=E\left(g\left(X_{6}\right)\right)+E\left(\int_{\tau_{X_{3}}}^{\tau_{X_{4}}}(f(x)) d t\right)+E\left(\int_{\tau_{X_{5}}}^{\tau_{X_{6}}}(f(x)) d t\right)-E\left(c\left(X_{4}\right) h_{y}\right)
\end{gathered}
$$

If this procedure is repeated with $X_{2}$ the solution at point $X_{1}$ can be written

$$
\begin{gathered}
u\left(X_{1}\right)=E\left(g\left(X^{\tau_{x}}\right)\right)+E\left(\int_{0}^{\tau_{x}}(f(x)) d t\right)=E\left(u\left(X_{2}\right)\right)+E\left(\int_{\tau_{X_{1}}}^{\tau_{X_{2}}}(f(x)) d t\right)= \\
=E\left(u\left(X_{3}\right)\right)-E\left(c\left(X_{3}\right) h_{x}\right)+E\left(\int_{\tau_{X_{1}}}^{\tau_{X_{2}}}(f(x)) d t\right)= \\
=E\left(g\left(X_{6}\right)\right)+E\left(\int_{\tau_{X_{1}}}^{\tau_{X_{2}}}(f(x)) d t\right)+E\left(\int_{\tau_{X_{3}}}^{\tau_{X_{4}}}(f(x)) d t\right) \\
+E\left(\int_{\tau_{X_{5}}}^{\tau_{X_{6}}}(f(x)) d t\right)-E\left(c\left(X_{4}\right) h_{y}\right)-E\left(c\left(X_{3}\right) h_{x}\right)
\end{gathered}
$$


In a general case Itô's formula can be written as

$$
u(\bar{x})=E\left(g\left(X^{\tau_{x}}\right)\right)+E\left(\int_{0}^{\tau_{x}}(f(x)) d t\right)+E\left(-\sum_{i} \frac{\partial u}{\partial n}\left(X_{i}\right) h_{n}\right)
$$

where

$E\left(g\left(X^{\tau_{x}}\right)\right)$ is the contribution of Dirichlet boundary conditions

$E\left(\int_{0}^{\tau_{x}}(f(x)) d t\right)$ is the contribution of the input function

$E\left(-\sum_{i} \frac{\partial u}{\partial n}\left(X_{i}\right) h_{n}\right)$ is the contribution of Neumann boundary conditions

In these expressions $X_{i}$ are the points where the particle reaches a Dirichlet boundary and $h_{n}$ is a small value such that Taylor's expansions are accurate enough.

It is clear that for a unique simulation Neumann boundaries can be reached several times.

To explain more clearly the general technique we propose the following academic example.

$$
\frac{\partial^{2} u}{\partial x^{2}}+3 \frac{\partial^{2} u}{\partial y^{2}}=-16
$$

with a rectangular boundary $D=(0,1) x(0,1)$ and mixed boundary conditions.

$$
\begin{array}{ll}
\frac{\partial u}{\partial x}=\beta(y) & x=0 \\
u=0 & x=1 \\
\frac{\partial u}{\partial y}=\alpha(x) & y=0 \\
u=\gamma(x) & y=1
\end{array}
$$

Itô's process is in this case:

$$
d \bar{X}=\bar{b}(x) d t+\overline{\bar{Z}}(x) d \bar{B}
$$

with $b_{i}=0, \sigma_{11}=2, \sigma_{12}=0, \sigma_{21}=0, \sigma_{22}=6$.

To determine the $\overline{\bar{Z}}$ matrix we use $\overline{\bar{C}}=\overline{\bar{Z}} * \overline{\bar{Z}}^{T}$, this is to say

$$
\left(\begin{array}{ll}
2 & 0 \\
0 & 6
\end{array}\right)=\left(\begin{array}{ll}
z_{11} & z_{12} \\
z_{21} & z_{22}
\end{array}\right)\left(\begin{array}{ll}
z_{11} & z_{12} \\
z_{21} & z_{22}
\end{array}\right)^{T}
$$

and therefore

$$
\overline{\bar{Z}}=\left(\begin{array}{cc}
\sqrt{2} & 0 \\
0 & \sqrt{6}
\end{array}\right)
$$

If we apply now Itô`s formula, the solution at point $(x, y)$ is

$$
u(\bar{x})=E\left(g\left(X^{\tau_{x}}\right)\right)+E\left(\int_{0}^{\tau_{x}}(f(x)) d t\right)
$$

In this example:

$$
\begin{gathered}
u(\bar{x})=E\left(g\left(X^{\tau_{x}}\right)\right)+E\left(\int_{0}^{\tau_{x}} 16 d t\right)=E\left(g\left(X^{\tau_{x}}\right)\right)+16 E\left(\int_{0}^{\tau_{x}} d t\right)= \\
=E\left(g\left(X^{\tau_{x}}\right)\right)+16 E\left(T_{x}\right)
\end{gathered}
$$


where:

- $E\left(g\left(X^{\tau_{x}}\right)\right)$ is the expected value of the particles positions when they reach for the first time a Dirichlet boundary condition.

- $E\left(T_{x}\right)$ is the expected value of the time the particles take to reach for the first time a Dirichlet boundary condition. In other words, the expected value of the particles stopping times.

Let a particle initially at position $X_{1}$ (see Figure 1). After a random walk as previously described the particle reaches the boundary at $X_{2}$ where a Neumann condition holds. Then the particle is reflected to $X_{3}$, and the process will be restarted at point $X_{3}=X_{2}+\left(h_{x}, 0\right)$. After the corresponding walk, the particle reaches the boundary at $X_{4}$ where again a Neumann condition holds. The particle is then reflected to $X_{5}=X_{4}+\left(0, h_{y}\right)$ and the process continues until the particle reaches a point in which a Dirichlet boundary condition holds $\left(X_{6}\right)$. Then the process stops.

Let:

$T_{1}$ the time it takes the particle to go from $X_{1}$ to $X_{2}$

$T_{2}$ the time it takes the particle to go from $X_{3}$ to $X_{4}$

$T_{3}$ the time it takes the particle to go from $X_{5}$ to $X_{6}$

By using Itô's formula, the contribution of this particle to the solution is

$$
u\left(X_{1}\right)=g\left(X_{2}\right)+16\left(T_{1}\right)=u\left(X_{2}\right)+16\left(T_{1}\right)
$$

In this expression $u\left(X_{2}\right)$ can be expanded as $u\left(X_{2}\right)=u\left(X_{3}\right)-\frac{\partial u}{\partial x}\left(X_{2}\right) h_{x}$ and therefore

$$
u=u\left(X_{2}\right)+16\left(T_{1}\right)=u\left(X_{3}\right)-\frac{\partial u}{\partial x}\left(X_{2}\right) h_{x}+16\left(T_{1}\right)
$$

but

and again

$$
u\left(X_{3}\right)=g\left(X_{4}\right)+16\left(T_{2}\right)=u\left(X_{4}\right)+16\left(T_{2}\right)
$$

$$
u\left(X_{4}\right)=u\left(X_{5}\right)-\frac{\partial u}{\partial y}\left(X_{4}\right) h_{y}
$$

and the above expression (44) becomes

$$
u=u\left(X_{3}\right)-\frac{\partial u}{\partial x}\left(X_{2}\right) h_{x}+16\left(T_{1}\right)=u\left(X_{5}\right)-\frac{\partial u}{\partial y}\left(X_{4}\right) h_{y}-\frac{\partial u}{\partial x}\left(X_{2}\right) h_{x}+16\left(T_{1}+T_{2}\right)
$$

Finally, as $X_{6}$ is a point in which a Dirichlet condition is stablished

$$
u\left(X_{5}\right)=g\left(X_{6}\right)+16\left(T_{3}\right)=0+16\left(T_{3}\right)
$$

and the contribution of this particles to the final solution is

$$
u=0-\frac{\partial u}{\partial y}\left(X_{4}\right) h_{y}-\frac{\partial u}{\partial x}\left(X_{2}\right) h_{x}+16\left(T_{1}+T_{2}+T_{3}\right)
$$

\subsection{Simulation procedures}

The partial differential equation to solve is:

$$
\mathrm{L} u=-f(x)
$$

with the following boundary conditions: 


$$
\begin{gathered}
u(x)=g(x) \text { si } x \in \partial D_{d} \\
\frac{\partial u(x)}{\partial n}=t(x) \text { si } x \in \partial D_{n}
\end{gathered}
$$

with $\partial D_{d} \cup \partial D_{n}=\partial D$ and $D_{d} \cap \partial D_{n}=\emptyset$, being $\partial D$ the boundary of the integration domain $D$ and

$$
\mathrm{L}(.)=\sum b_{i} \frac{\partial(.)}{\partial x_{i}}+\frac{1}{2} \sum \sum \sigma_{i j} \frac{\partial^{2}(.)}{\partial x_{i} \partial x_{j}}
$$

As previously stated the solution is

$$
u(\bar{x})=E\left(g\left(X^{\tau_{x}}\right)\right)+E\left(\int_{0}^{\tau_{x}}(f(x)) d t\right)+E\left(-\sum_{i} \frac{\partial u}{\partial n}\left(X_{i}\right) h_{n}\right)
$$

Then the simulation procedure is as follows:

3.3.1 Determine the Ito's process $b_{i}$ y $\sigma_{i j}$ coefficients:

$$
\begin{gathered}
d \bar{X}=\bar{b}(x) d t+\overline{\bar{Z}}(x) d \bar{B} \\
\left(\begin{array}{c}
d x_{1} \\
\vdots \\
d x_{n}
\end{array}\right)=\left(\begin{array}{c}
b_{1} \\
\vdots \\
b_{n}
\end{array}\right) d t+\left(\begin{array}{ccc}
Z_{11} & \ldots & Z_{1 n} \\
\vdots & \ddots & \vdots \\
Z_{n 1} & \ldots & Z_{n n}
\end{array}\right)\left(\begin{array}{c}
d B_{1} \\
\vdots \\
d B_{n}
\end{array}\right)
\end{gathered}
$$

$\overline{\bar{Z}}(x)$ is defined as the matrix calculated from the equation

$$
\overline{\bar{C}}=\overline{\bar{Z}} * \overline{\bar{Z}}^{T}
$$

where the parameters $\sigma_{i j}$ are the elements of the $\overline{\bar{C}}$ matrix and the parameters $b_{i}$ are the elements of the $\bar{b}(x)$ vector.

3.3.2 Set the $s$ and $h$ values, where $s^{2}$ is the variance of the Brownian motion process and $h$ the increment to use in the Taylor series expansions.

The Brownian motion variance will determine the length of every leap of a particle for every time step.

The following figures show some examples of a Brownian motion process going on from the same point $(\mathrm{x}=0.6, \mathrm{y}=2.7)$ with Dirichlet boundary condition at $\mathrm{x}=0$ and Neumann condition at the reflecting border (Figures 2 and 3).

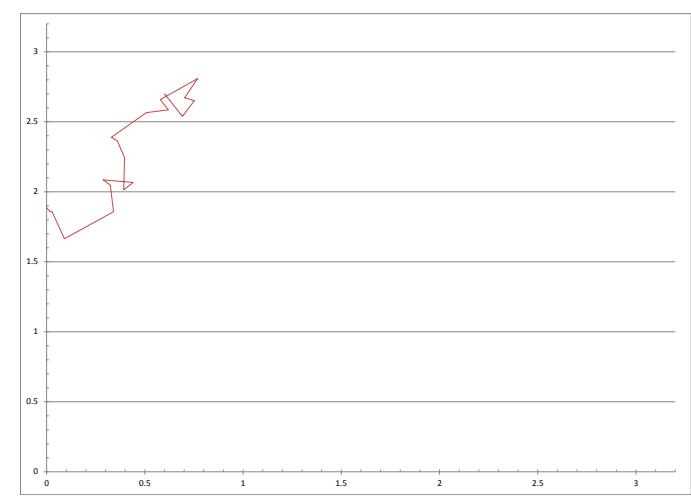

Standard deviation 0.1

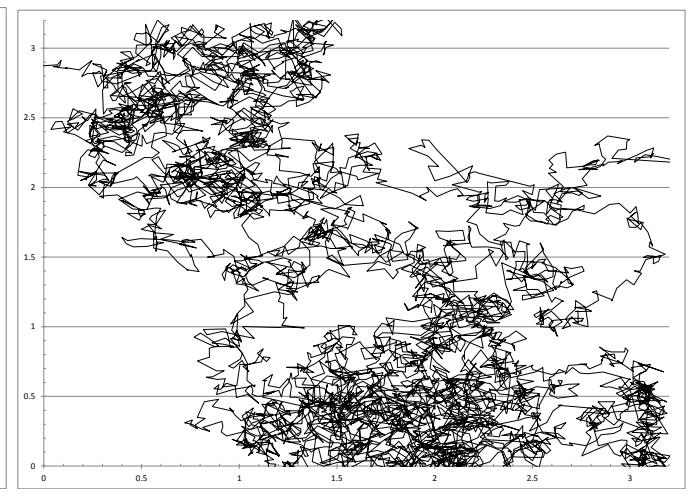

Standard deviation 0.05

Figure 2. Examples of paths for different values of the BM standard deviation 


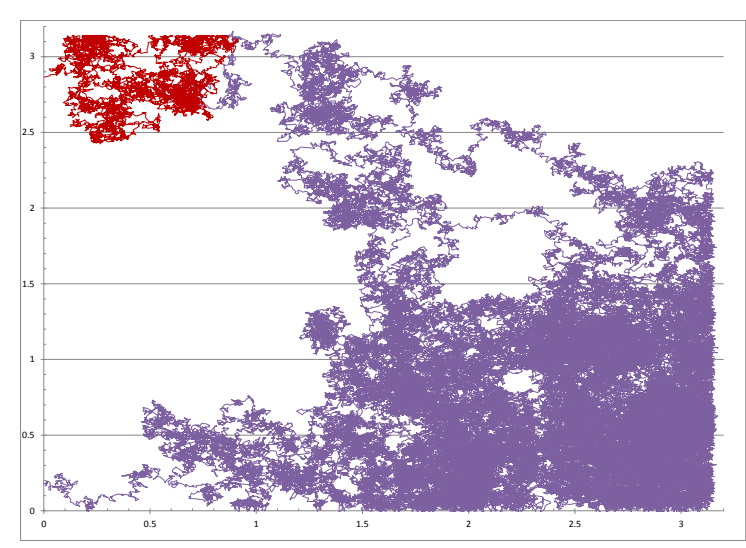

Figure 3. Two BM examples starting from the same point with the same standard deviation 0,01

A high value of the variance $s^{2}$ means a faster simulation process but it is generally less exact if the boundaries lie near the particle.

The $h$ value has to be small enough to fulfill the Taylor expansion conditions but large enough to guarantee that the particle is always inside the domain of integration. In this paper a value of $h$ five times the standard deviation of the Brownian motion process has been used, this is to say

$$
h=5 \sqrt{\Delta t}=5 s
$$

\subsubsection{Obtain the solution in $\bar{x}$.}

To obtain the problem solution we must calculate

$$
u(\bar{x})=E\left(g\left(X^{\tau_{x}}\right)\right)+E\left(\int_{0}^{\tau_{x}}(f(x)) d t\right)+E\left(-\sum_{i} \frac{\partial u}{\partial n}\left(X_{i}\right) h_{n}\right)
$$

A possibility is to use Montecarlo method: we select a large number of particles for each point in which the solution must be evaluated.

From $\bar{x}$, every one of these particles performs, for each $\Delta t$, a random step defined by Itô's process, namely

$$
d \bar{X}=\bar{b}(x) d t+\overline{\bar{Z}}(x) d \bar{B}
$$

where $d B$ is a standard Brownian motion with Gaussian distribution $N\left(0, s^{2}\right)$

Remember that the time spent for each particle to reach a boundary in which a Dirichlet condition holds, $\tau_{x}$, is called stopping time.

The contribution of a particle to the solution is

$$
u_{j}=\frac{\left(g\left(X^{\tau_{x}}\right)\right)+\left(\int_{0}^{\tau_{x}}(f(x)) d t\right)+\left(-\sum_{i} \frac{\partial u}{\partial n}\left(X_{i}\right) h_{n}\right)}{N}
$$

where $i$ is the number of times the particle reaches a boundary with Neumann condition, $N$ the number of particles used in the simulation and $\tau_{x}$ the time spent for each particle to reach a boundary in which a Dirichlet condition holds (the so called stopping time). In equation (58):

- $g\left(X^{\tau_{x}}\right)$ is the contribution of Dirichlet type boundary conditions. It is evaluated as the value of the boundary condition at the point first reached by the particle.

- $\int_{0}^{\tau_{x}}(f(x)) d t$ is the contribution of the input function at the solution and must be evaluated until the stopping time. 
- $\quad-\sum_{i} \frac{\partial u}{\partial n}\left(X_{i}\right) h_{n}$ is the contribution of Neumann type boundary conditions. A particle may reach a Neumann type condition never, once or more times before reaching a point in which a Dirichlet boundary condition holds.

The final solution estimation is:

$$
u=\sum_{j=1}^{N} u_{j}
$$

\subsection{Two dimensional examples}

3.4.1 Let $\Omega$ be the unit circle defined as $x^{2}+y^{2} \leq 1$ in Cartesian coordinates. We state the following differential operator.

$$
\Delta U=0
$$

with boundary conditions

$$
\begin{gathered}
U(r, 0)=U(r, 2 \pi)=0 \\
U(1, \theta)=\sin \left(\frac{\theta}{2}\right), \quad 0<\theta<2 \pi
\end{gathered}
$$

The analytical solution of this problem is $U=\sin \left(\frac{\theta}{2}\right) \sqrt{r}$ (See Figure 4)

The Itô's process governing the particles motion is

$$
d \bar{X}=\bar{b}(x) d t+\overline{\bar{Z}}(x) d \bar{B}
$$

In this case $b_{i}=0, \sigma_{i, j}=1 \forall i=j$ and $\sigma_{i, j}=0 \forall i \neq j$. Therefore, as $\overline{\bar{C}}=\overline{\bar{Z}} * \overline{\bar{Z}}^{T}$ and $\overline{\bar{C}}=\left(\begin{array}{ll}1 & 0 \\ 0 & 1\end{array}\right)$, it results that $\overline{\bar{Z}}=\left(\begin{array}{ll}1 & 0 \\ 0 & 1\end{array}\right)$.

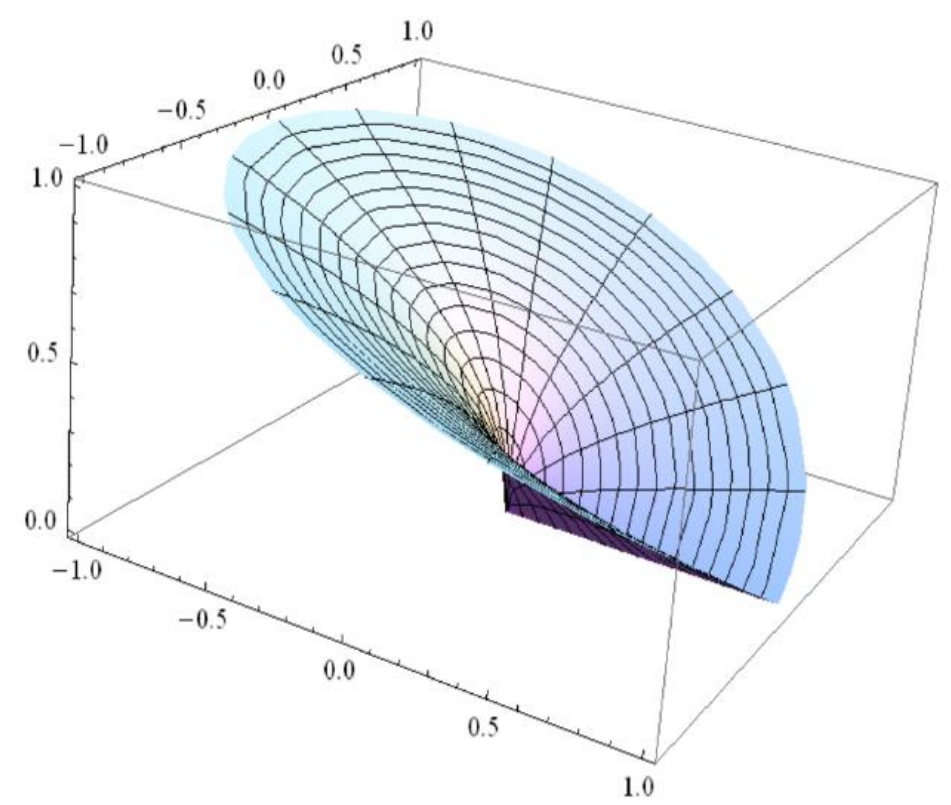

Figure 4. Analytical solution of the problem stated in section 3.4.1

It follows that $d x_{1}=d B_{1}$ and $d x_{2}=d B_{2}$

The numerical solution is: 


$$
u(\bar{x})=E\left(g\left(X^{\tau_{x}}\right)\right)+E\left(\int_{0}^{\tau_{x}}(f(x)) d t\right)
$$

because in this example there are not Neumann boundary conditions. In this case $f(x)=0$, and therefore

$$
u(\bar{x})=E\left(g\left(X^{\tau_{x}}\right)\right)
$$

where $\tau_{x}$ is the stopping time of each particle.

Figure 5 shows the comparison between the exact (analytical) solution and the simulation for different points of the integration domain.

For radii in the [0.3 al 0.9] interval 5.000 particles have been generated with a process standard deviation of 0.01 .

For 0.2 and 0.1 radii 15.000 particles have been generated with a process standard deviation of 0.001 .

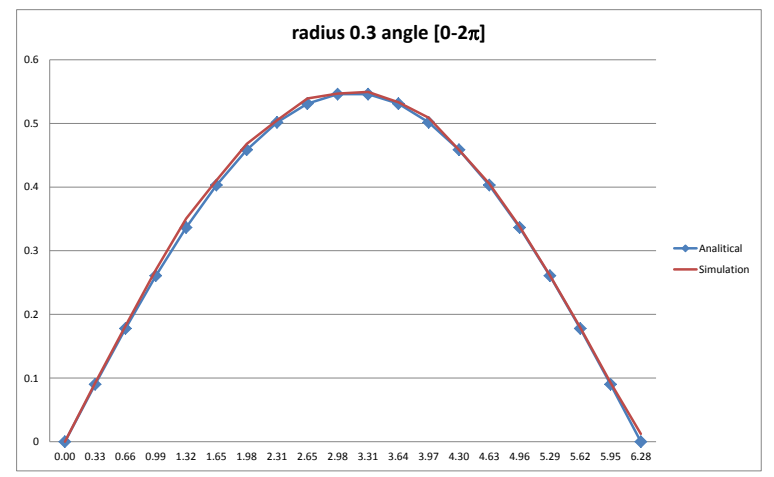

a) $r=0.3, \theta$ in $[0,2 \pi]$

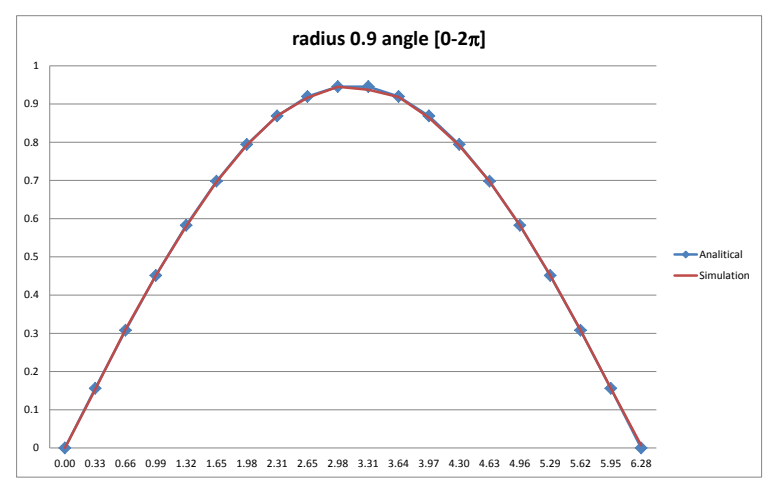

a) $\mathrm{r}=0.9, \theta$ in $[0,2 \pi]$

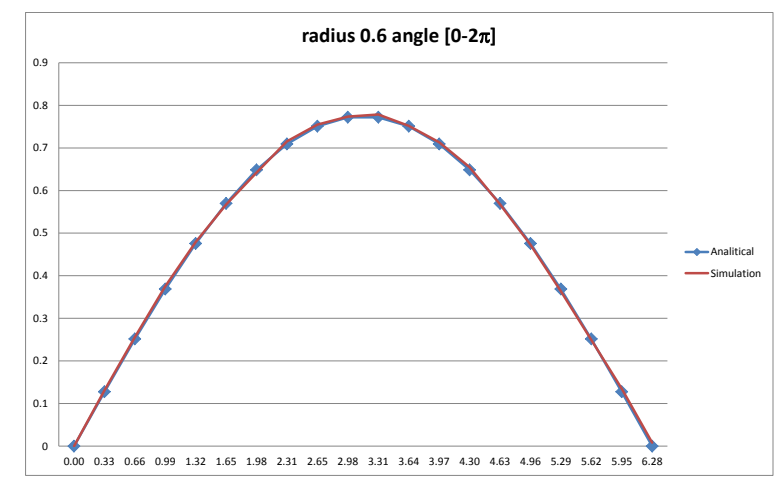

b) $r=0.6, \theta$ in $[0,2 \pi]$

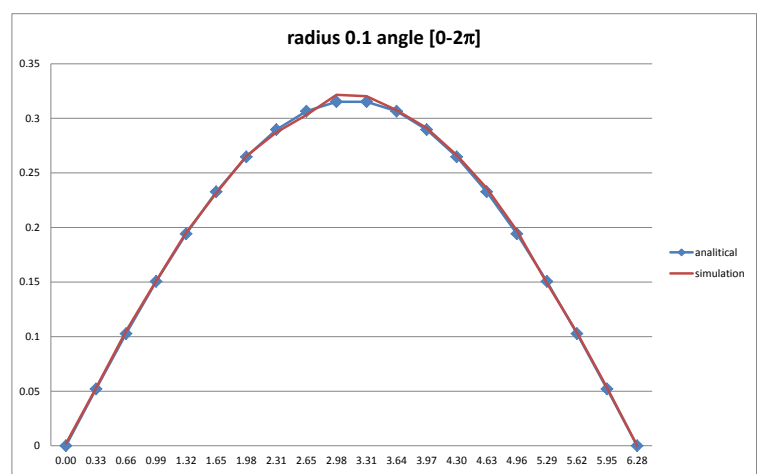

b) $r=0.1, \theta$ in $[0,2 \pi]$

Figure 5. Simulation vs. analytical solutions of problem 3.4.1 for different domain points

3.4.2 Consider the following problem, in which both Dirichlet and Neumann boundary conditions are defined:

$$
\Delta U=0, \quad 0 \leq X \leq \pi \quad 0 \leq Y \leq \pi
$$

with boundary conditions 


$$
\begin{gathered}
u(x=0, y)=-\frac{1}{\tanh (\pi)}[\cosh (y)-\tanh (\pi) \operatorname{senh}(y)] \\
\left.\frac{\partial u}{\partial x}\right|_{(\pi, y)}=0 ; \quad 0 \leq y \leq \pi \\
\left.\frac{\partial u}{\partial y}\right|_{(x, 0)}=\cos (x) \\
\left.\frac{\partial u}{\partial y}\right|_{(x, \pi)}=0 \quad 0 \leq x \leq \pi
\end{gathered}
$$

The analytical solution, shown in Figure 6, is:

$$
\mathrm{u}(\mathrm{x}, \mathrm{y})=-\frac{1}{\tanh (\pi)}[\cosh (y)-\tanh (\pi) \operatorname{senh}(y)] \cos (x)
$$

The Itô's process governing the particles motion is

$$
d \bar{X}=\bar{b}(x) d t+\overline{\bar{Z}}(x) d \bar{B}
$$

Therefore $d x_{1}=d B_{1}$ and $d x_{2}=d B_{2}$

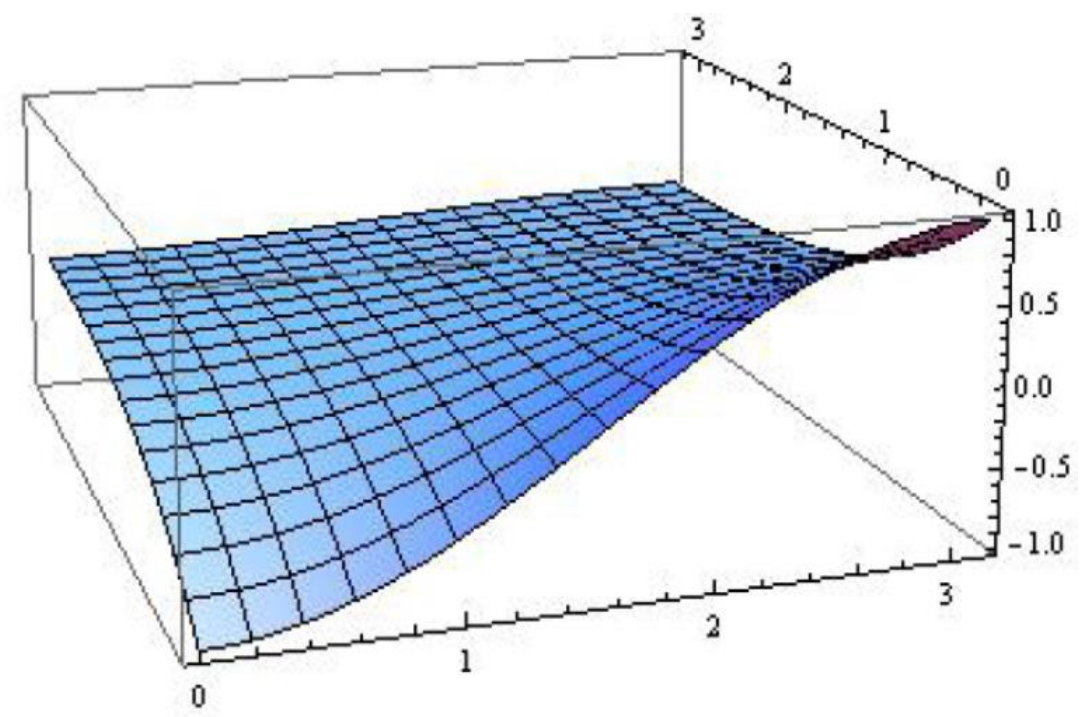

Figure 6. Analytical solution of problem stated in section 3.4.2

The simulation solution is

$$
u(\bar{x})=E\left(g\left(X^{\tau_{x}}\right)\right)+E\left(\int_{0}^{\tau_{x}}(f(x)) d t\right)+E\left(-\sum_{i} \frac{\partial u}{\partial n}\left(X_{i}\right) h_{n}\right)
$$

because in this case $f(x)=0$

15.000 particles have been generated for each point, the $\mathrm{h}$ value is 0.05 and the standard deviation of the Brownian motion is 0.01 .

Figure 5 shows the comparison between the exact (analytical) solution and the simulation for different points of the integration domain. 


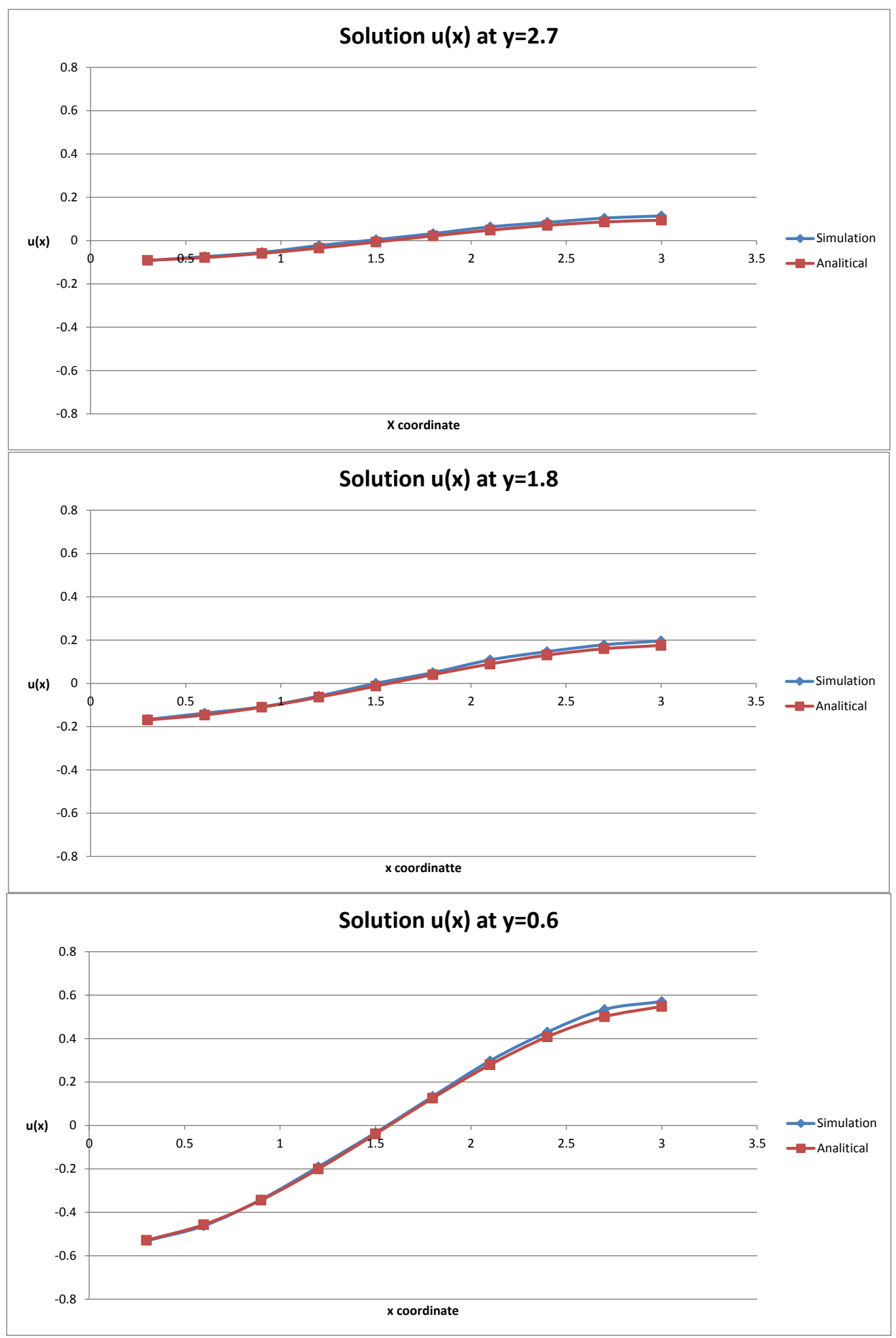

Figure 7. Simulation vs. analytical solutions of problem 3.4.2 for different domain points

\section{PARABOLIC PROBLEM GENERAL APPROACH}

Consider the following partial differential equation:

$$
\mathrm{L}(u)=-f(\bar{x}, t)
$$

with boundary and initial conditions: 


$$
\begin{gathered}
u(\bar{x}, t)=g(\bar{x}, t) \text { si } \bar{x} \in \partial D_{d} \\
\frac{\partial u(\bar{x}, t)}{\partial n}=c(\bar{x}, t) \text { if } \bar{x} \in \partial D_{n} \\
u(\bar{x})=l(\bar{x}) \text { if } t=0
\end{gathered}
$$

with $t>0, \bar{x} \in D, \partial D_{d} \cup \partial D_{n}=\partial D$ and $D_{d} \cap \partial D_{n}=\emptyset$, being $\partial D$ the boundary of the integration domain $D$. The $\mathrm{L}($.$) operator can be written as$

$$
\mathrm{L}(.)=-\frac{\partial(.)}{\partial t}+\sum b_{i} \frac{\partial(.)}{\partial x_{i}}+\frac{1}{2} \sum \sum \sigma_{i j} \frac{\partial^{2}}{\partial x_{i} \partial x_{j}}
$$

where the time $t$ may be considered as a new coordinate in the process.

In the n-dimensional case

$$
d \bar{X}=\bar{b}(x) d t+\overline{\bar{Z}}(x) d \bar{B}
$$

that can also be written as

$$
\left(\begin{array}{c}
d x_{0} \\
d x_{1} \\
\vdots \\
d x_{n}
\end{array}\right)=\left(\begin{array}{c}
-1 \\
b_{1} \\
\vdots \\
b_{n}
\end{array}\right) d t+\left(\begin{array}{ccc}
0 & \cdots & 0 \\
Z_{11} & \cdots & Z_{1 n} \\
\vdots & \ddots & \vdots \\
Z_{n 1} & \cdots & Z_{n n}
\end{array}\right)\left(\begin{array}{c}
d B_{0} \\
d B_{1} \\
\vdots \\
d B_{n}
\end{array}\right)
$$

where $x_{0}$ is the time coordinate and $x_{i,}, i \in(0, n)$ the spatial coordinates.

At every time interval $d t$, the particle moves randomly in the space $\left(x_{i}\right)$ and goes back a fixed time increment $d t$.

If the particle reaches a Neumann boundary condition, the particle bounces back and continues the random motion inside the integration domain.

The initial condition $\mathrm{u}(\overline{\mathrm{x}})=\mathrm{l}(\overline{\mathrm{x}})$ if $\mathrm{t}=0$ is, in this process, considered as a Dirichlet boundary condition.

The particle random motion may stop because:

- Particle reaches a Dirichlet boundary in the space $\left(x_{i}\right)$

- Particle reaches time $\mathrm{t}=0$, because, as it was stated before, this initial condition works as a Dirichlet Condition for this simulation process.

\subsection{Two dimensional parabolic example}

To explain more clearly the general technique we propose the following academic example:

The PDE to solve is:

$$
\frac{\partial u}{\partial t}=\left(\frac{\partial^{2} u}{\partial x^{2}}+\frac{\partial^{2} u}{\partial y^{2}}\right)
$$

with integration domain $0 \leq x \leq 2,0 \leq y \leq 4, t \geq 0$ (see Figure 8) and boundary conditions

$$
\begin{gathered}
\frac{\partial u(x=0, y, t)}{\partial x}=0, \quad u(x, y=0, t)=0 \\
\frac{\partial u(x=2, y, t)}{\partial x}=0, \quad u(x, y=4, t)=0
\end{gathered}
$$


and the inital condition

$$
u(x, y, t=0)=7
$$

$$
u(x, y=4, t)=0
$$

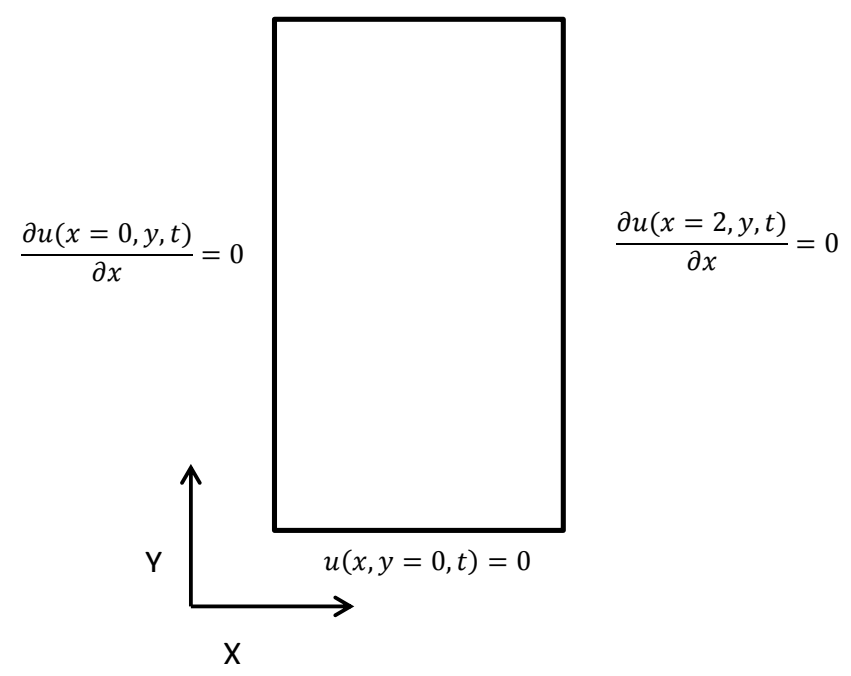

Figure 8. Integration domain for the problem presented in section 4.1

The analytical solution for this problem is:

$$
u(x, y, t)=\frac{14}{\pi} \sum_{n=1}^{\infty}(1-\cos (n \pi))\left(\frac{1}{n}\right) e^{\left(\frac{-t \pi^{2} n^{2}}{16}\right)} \sin \left(\frac{n \pi y}{4}\right)
$$

The stochastic process approach is

$$
\begin{gathered}
\left(\frac{\partial^{2} \mathrm{u}}{\partial \mathrm{x}^{2}}+\frac{\partial^{2} \mathrm{u}}{\partial \mathrm{y}^{2}}\right)-\frac{\partial \mathrm{u}}{\partial \mathrm{t}}=0 \\
L(.) u(x))-f(x) \\
f(x)=0 \\
L(.)=\sum_{i} b_{i} \frac{\partial .}{\partial x_{i}}+\frac{1}{2} \sum_{i j} \sigma_{i j} \frac{\partial^{2}}{\partial x_{i} \partial x_{j}}
\end{gathered}
$$

In this case $i=0,1,2$ y $j=0,1,2$.

The Itô process can be written as

$$
d \bar{X}=\bar{b}(x) d t+\overline{\bar{Z}}(x) d \bar{B}
$$

or

$$
\left(\begin{array}{l}
d x_{0} \\
d x_{1} \\
d x_{2}
\end{array}\right)=\left(\begin{array}{c}
-1 \\
0 \\
0
\end{array}\right) d t+\left(\begin{array}{ccc}
0 & 0 & 0 \\
0 & \sqrt{2} & 0 \\
0 & 0 & \sqrt{2}
\end{array}\right)\left(\begin{array}{l}
d B_{0} \\
d B_{1} \\
d B_{2}
\end{array}\right)
$$

with $d B=N(0, d t)$. 
The estimate of the solution is

$$
u(\bar{x})=E\left(g\left(X^{\tau_{x}}\right)\right)+E\left(\int_{0}^{\tau_{x}}(f(x)) d t\right)+E\left(-\sum_{i} \frac{\partial u}{\partial n}\left(X_{i}\right) h_{n}\right)
$$

The second and third addends are null because $f(x)=0$ and all Neumann boundary conditions are also null.

Then

$$
u(\bar{x})=E\left(g\left(X^{\tau_{x}}\right)\right)
$$

where $\tau_{x}$ is the already defined stopping time.

Suppose we want to calculate the problem solution at a point with coordinates $(x, y, t)$. As always, the simulation solution is to generate a number of particles at this point and apply the above described technique..

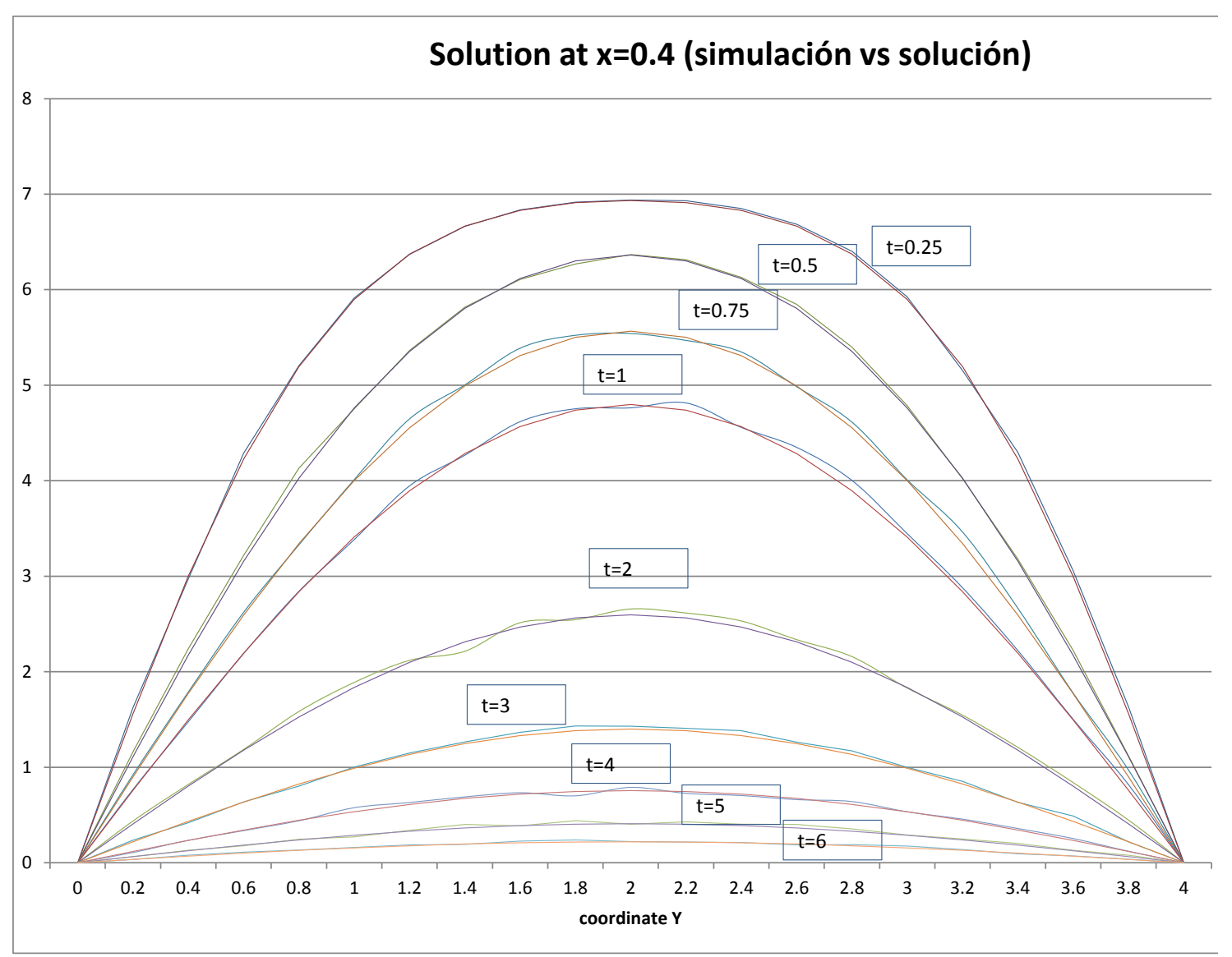

Figure 9. Solution of the problem proposed in section 4.1

\section{CONCLUSIONS}

A simulation stochastic method to solve partial differential equations (of elliptic and parabolic type) has been presented. The analysis of the specific boundary and (in the parabolic type) initial conditions has been carried out. A number of academic examples, with known analytical solutions, have been used as benchmarks, and the simulation solution has agreed very well with the exact solutions in all cases.

The main advantages of this technique are:

- No domain discretization is necessary. 
- No need for a solver, because there are not systems of equations in the solution process

- It is not necessary to solve the PDE in the whole integration domain. The solution can be obtained only where it is needed (a point, several points, a particular area of the domain).

- In parabolic problems, to obtain the solution at time $T_{f}$ it is not necessary to obtain it at intermediate times $\left(T_{0} ; T_{f}\right)$.

- The approximation error is a function of the number of particles generated in the simulation, and decreases as the number of particles increases. This is so because the solution is an expected value, and because of the central limit theorem the variance will decrease as the number of elements (particles) increases.

- The speed of the process increases as the variance of the stochastic process (Itô's process) increases. However, near the boundaries, the variance should be kept small enough as the walk does not go outside the domain

- As the solution estimate is an expected value, parallel methods may be applied in a very easy way. For instance, the same accuracy will be obtained with a CPU generating a 10.000 particles simulation as with 100 CPU's generating a 100 particles simulation each. As the only process that can be considered time consuming is the particles generation and the corresponding random walk, the process time can be directly divided by the number of CPU's in the cluster.

\section{AKNOWLEGMENTS}

The authors acknowledge the financial support provided by Grant GRC2014/039, "Axudas para a consolidación e estruturación de unidades de investigación competitivas do Sistema Universitario de Galicia do 2014: Grupo de Referencia Competitiva" of the Xunta de Galicia (Spain) and by the Research Group of Numerical Methods in Engineering of the University of A Coruña.

\section{REFERENCES}

[1] Ritz, Walter, Über eine neue Methode zur Lösung gewisser Variationsprobleme der mathematischen Physik, Journal für die Reine und Angewandte Mathematik, vol. 135, pages 1-61, 1909.

[2] Øksendal, Bernt, Stochastic Differential Equations. An Introduction with Applications, 5th edition, corrected 2nd printing. Springer Verlag, 2000.

[3] Kloeden, P.E., Platen, E., Numerical Solution of Stochastic Differential Equations, Volume 23 of the series Applications of Mathematics pp 103-160, Springer Verlag, 1992

[4] Itô, Kiyosi, Stochastic Integrals. Proc. Imperial Acad. Tokyo, 20, 519-524, 1944.

[5] Itô, Kiyosi, On stochastic differential equations. Memoirs, American Mathematical Society 4, 1-51, 1951.

[6] Carmona, Alexis, Ecuaciones diferenciales estocásticas. Thesis. Universidad de Buenos Aires, Argentina, 2009

[7] Karatzas, I., S. Steven, Brownian Motion and Stochastic Calculus (2nd ed.), Springer Verlag, 1991 
[8] Rincón, Luis, Construyendo la integral estocástica de Itô. Aportaciones Matemáticas, Series en Comunicaciones 35, 265-283, 2005.

[9] Itô, Kiyosi, On a formula concerning stochastic differentials, Nagoya Mathema-tical Journal 3: 55-65, 1951

[10] Chung, K.L., Williams, R.J., Introduction to Stochastic Integration, Springer Verlag, 2014

[11] Granero Belinchón, Rafael, Sobre algunas relaciones entre la probabilidad y las ecuaciones diferenciales, Thesis, Université Paris-Dauphine and Universidad Autónoma de Madrid, 2009

[12] Mascagni, Michael, Monte Carlo Methods for Partial Differential Equations. Florida State University, USA, 2011

[13] Grigoriu, Mircea, Stochastic Calculus, Applications in science and engineering. Birkhäuser, 2002 